\title{
Neural Ganglioside GD2+ Cells Define a Subpopulation of Mesenchymal Stem Cells in Adult Murine Bone Marrow
}

\author{
Jie Xua,c WenJun Fan ${ }^{a, c}$ Xi Xiang Tu ${ }^{a}$ Teng Zhang ${ }^{a}$ Zhi Jie Hou ${ }^{a}$ Tao Guo \\ Xin Shu ${ }^{a}$ Xi Luo ${ }^{a}$ Yang Liu ${ }^{a}$ Fei Peng ${ }^{a}$ Chang Wang ${ }^{a}$ LingZhi Xu $^{a}$ Han Zhou \\ Quentin Liu ${ }^{\mathrm{a}}$
}

aInstitute of Cancer Stem Cell, DaLian Medical University Cancer Center, Liaoning, China; ${ }^{b}$ Yale Stem Cell Center, Yale University School of Medicine, New Haven, CT, USA; 'These authors contribute equally to this work

\section{Key Words}

Murine Bone marrow • Mesenchymal stem cells • Neural ganglioside GD2 • Identification

\begin{abstract}
Background/Aims: Due to the lack of specific markers, the isolation of pure mesenchymal stem cells (MSCs) from murine bone marrow remains an unsolved problem. The present study explored whether the neural ganglioside GD2 could serve as a single surface marker to uniquely distinguish murine bone marrow MSCs (mBM-MSCs) from other marrow elements. Methods: Immunocytochemistry and flow cytometry, in combination with quantitative RT$\mathrm{PCR}$, were used to identify the expression of GD2 on culture-expanded mBM-MSCs. GD2 ${ }^{+}$and GD2 fractions from mBM-MSCs cultures were sorted by immunosorting. Flow cytometry was performed to further analyze the biomarkers of GD2-sorted and unsorted cells. Employing CFU-F assay and CCK-8 assay, we examined the clonogenic and proliferative capabilities of GD2sorted and unsorted cells. Using oil red $\mathrm{O}$ and von Kossa staining assay, we also assessed the multi-lineage potential of GD2-sorted and unsorted cells. Results: We found that mBM-MSCs expressed a novel surface marker the neural ganglioside GD2. Importantly, mBM-MSCs were the only cells within bone marrow that expressed this marker. Further studies demonstrated that a homogenous population of MSCs could be obtained from bone marrow cultures in early passages by GD2 immunosorting. Compared to parental cells, GD2+-sorted cells not only possessed much higher clonogenic and proliferative capabilities but also had significantly stronger differentiation potential to adipocytes and osteoblasts. Furthermore, GD2+-sorted cells displayed enhanced expression of ES markers SSEA-1 and Nanog. Conclusion: Our observations provide the first demonstration that GD2 may serve as a maker for identification and purification of mBM-MSCs. Meanwhile, our study indicates that the cells selected by GD2 are a subpopulation of MSCs with features of primitive precursor cells.
\end{abstract}

Copyright (C) 2013 S. Karger AG, Basel 
Xu et al.: mBM-MSCs Express the Neural Ganglioside GD2

\section{Introduction}

In recent years, mesenchymal stem cells (MSCs) with their capability of self-renewal and multi-differentiation potential have been considered as an ideal source for cell and gene therapy strategies [1-3]. However, before MSCs application in humans, the extensive preclinical studies must be carried out in animal models to confirm their safety and efficacy [4, 5]. Many pre-clinical studies have been carried out in the mouse [6-8], which is a widely used mammalian physiological and pathophysiological model. MSCs isolation from human bone marrow is classically performed by adhesion to plastic [9]. However, this standard method is not suitable for the isolation of murine bone marrow MSCs (mBM-MSCs). Compared to human BM-MSCs, murine BM-MSCs are more readily contaminated by other bone marrowderived adherent cells such as fibroblasts, macrophages or hematopoietic cells [10-12]. In addition, no specific marker has been reported for mBM-MSCs, although a combination of expressing Sca-1 and CD44 and not expressing CD45 and CD11b has been used to identify mBM-MSCs [13-15]. Thus the mBM-MSCs identified using the currently methods most likely represent a heterogeneous cell population, which will prohibit further studying their therapeutic potential and complicate data interpretation. Therefore, there is an urgent need for novel markers and methods to isolate a pure MSCs population from mouse bone marrow. Recently a novel surface marker neural ganglioside GD2 has been reported to be expressed on human BM- MSCs, both in vivo and following expansion in culture. Importantly, MSCs are the only cells within the normal marrow that express this marker, which appears to be the first reported single surface marker that uniquely distinguishes MSCs from other marrow elements [16]. Moreover, our recent study has demonstrated the expression of GD2 in cultured MSCs from umbilical cord (UC-MSCs), suggesting that GD2 can be used for the identification and purification of human UC-MSCs [17].

Here, we reported that BM-MSCs from various murine strains expressed GD2, while fibroblasts and macrophages did not. On the basis of these observations, we established a novel and efficient method to isolate a homogenous cell population of BM-MSCs from the heterogeneous mixture of murine bone marrow cells using GD2 immunosorting and further analyzed the phenotypic and functional characteristics of this cell population.

\section{Materials and Methods}

Cell isolation and culture

All experiments were performed under institutionally approved protocols for the use of animals in research. BM samples were obtained from 3 inbred strains of mice (Institute of Experimental Animals, Beijing, China): C57BL/6, BALB/c and FVB/N and mBM-MSC were collected from at least 3 mice of each strain. Male mice 2 to 8 weeks old were used, and they were individually killed by CO2. mBM-MSCs were isolated according to a protocol modified from Kopen et al. [18]. Briefly, bone marrow was collected by flushing femurs and tibias with growth medium, which consisted of DMEM/DF12 medium (Gibco) and $10 \%$ fetal bovine serum (HyClone), supplemented with $10 \mathrm{ng} / \mathrm{mL}$ vascular endothelial growth factor (VEGF; Sigma), 10ng/mL epidermal growth factor (EGF; Sigma), 100U penicillin/streptomycin (Sigma) and 2mM L-glutamine (Gibco). Cells were then plated at a density of $2 \times 10^{6}$ cells $/ \mathrm{cm}^{2}$ in non-coated T-25 or T-75 cell culture flasks (Beckon Dickinson). After 3 days, the medium was replaced and non-adherent cells were removed. Once $90 \%$ confluence had been reached, adherent cells were harvested by $0.25 \%$ trypsin-EDTA (Gibco), and then replated. The medium was changed every 3-4 days.

Bone marrow-derived macrophages from 4-week-old C57BL/6 mice were prepared as described previously [19]. Mouse fibroblasts were isolated from skin of 1-day-old C57BL/6 mice as reported previously [20]. A melanoma cell line was cultured in DMEM (Gibco) with 20\% FBS (HyClone), serving as the positive control.

Cell separation

mBM-MSCs from 4-week-old C57BL/6 mice (P2) were incubated with murine monoclonal antibody against GD2 (clone 14.G2A, BD Biosciences) for $30 \mathrm{~min}$ at $4^{\circ} \mathrm{C}$. Then cells were incubated with beads 
Xu et al.: mBM-MSCs Express the Neural Ganglioside GD2

Table 1. Reverse transcription-polymerase chain reaction primer sequences information

\begin{tabular}{|c|c|c|c|c|}
\hline \multirow[b]{2}{*}{ Primer } & \multicolumn{2}{|c|}{ Primer pair sequence } & \multirow{2}{*}{$\begin{array}{l}\text { Annealing } \\
\text { temperature, }{ }^{\circ} \mathrm{C}\end{array}$} & \multirow{2}{*}{$\begin{array}{l}\text { Product } \\
\text { size, bp }\end{array}$} \\
\hline & Forward & Reverse & & \\
\hline$\beta$-actin & CTGTCCCTGTATGCCTCTG & ATGTCACGCACGATTTCC & 60 & 200 \\
\hline GD2 synthase & CTGAGGGCTGTTTCTGTCGC & GGGTAGGGATGATGGTGGGT & 60 & 474 \\
\hline Adipsin & GTACTTCGTGGCTCTGGTGATC & CCACTTCTTTGTCCTCGTATTGC & 60 & 416 \\
\hline LPL & GCCTGAGTTGTAGAAAGAATCGC & TCTCCCTAGCACAGAAGATGACC & 55 & 441 \\
\hline CBFA1 & CCGCACGACAACCGCACCAT & CGCTCCGGCCCACAAATCTC & 55 & 289 \\
\hline Collagen I & GAAGTCAGCTGCATACAC & AGGAAGTCCAGGCTGTCC & 55 & 313 \\
\hline $\mathrm{OC}$ & TCTGCTCACTCTGCTGAC & GGAGCTGCTGTGACATCC & 55 & 388 \\
\hline
\end{tabular}

coated with goat anti-mouse IgG microbeads (mini-Macs, Miltenyi Biotec) according to the manufacturer's instructions. Cell suspensions were separated by using a magnetic separation column (Miltenyi Biotec).The uncoated cells (GD2 ${ }^{-}$cells) were removed by several washes with PBS/BSA. The retained cells were termed the $\mathrm{GD}^{+}$cells.

\section{Flow cytometry}

Abs were obtained from BD Biosciences unless stated otherwise. mBM-MSCs (n=3, p2) obtained from 3 different mouse strains aged 4 weeks old were respectively stained with murine monoclonal antibody against GD2 and FITC-conjugated goat anti-mouse secondary antibody. Staining without the primary antiGD2 antibody served as a negative control. Using the same methods, the analysis was performed on mBMMSCs from 2- to 8-week-old C57BL/6 mice. GD2 ${ }^{+}$, GD2 ${ }^{-}$or unsorted cells from 4-week-old C57BL/6 mice were respectively stained with PE-conjugated antibodies against CD45, CD11b, Sca-1, CD105 and SSEA1, or FITC-conjugated antibodies against CD34 and C-kit. Rat isotypic antibodies served as the control. In addition, $\mathrm{GD}^{+}, \mathrm{GD}^{-}$and unsorted cells were respectively stained with rabbit anti-Nanog antibody and FITC-conjugated goat anti-rabbit secondary antibody (Chemicon). Staining without the primary anti- Nanog antibody served as a negative control. Cells were stained in single label and then analyzed by flow cytometry with a FACScan (Becton Dickinson).

\section{Immunocytochemistry}

mBM-MSCs (P2), fibroblasts, macrophages and melanoma cells were cultured on the sterile chamber slides. After 2 days, cells were fixed in $4 \%$ paraformaldehyde (Sigma) and were incubated with primary antibodies against GD2.The reaction was visualized with a biotinylated goat anti-mouse secondary antibody with $\mathrm{ABC}$ substrate (Zymed). All slides were counterstained with hematoxylin for 2 minutes.

CFU-F assay

Equal number $\left(3 \times 10^{3}\right)$ of $\mathrm{GD}^{+}$, GD2 or unsorted cells were respectively seeded in $\mathrm{T}-25$ flasks. Medium was replaced every three days. After 14 days in culture, the flasks were washed twice, fixed with $100 \%$ methanol, and stained with 3\% Crystal violet. Three replicate plates were used for the experiments to obtain a mean value. Cell clusters consisting of at least 50 fibroblasts were scored as a CFU-F colony.

\section{Proliferation assay}

$\mathrm{GD}^{+}, \mathrm{GD} 2$ or unsorted cells were respectively seeded in triplicate in 96-well plates $\left(2 \times 10^{3}\right.$ cells/well). Three replicas were performed for each cell population. Ten microliters of sterile cell counting kit-8(cck-8) (Beyotime) were added to each well and incubated for $3 \mathrm{~h}$ at $37^{\circ} \mathrm{C}$. The viable cell numbers was determined every 3 days for 12 days. The optical density values were determined at least in triplicate against a reagent blank at a test wavelength of $450 \mathrm{~nm}$ and reference wavelength of $630 \mathrm{~nm}$.

\section{Multi-lineage differentiation}

In the same conditions, unsorted and $\mathrm{GD}^{+}$cells were induced to osteoblasts and adipocytes as previous described [11]. All the reagents for induction were purchased from Sigma. The adipocytes were stained with Oil Red $\mathrm{O}$ and the osteoblasts with von Kossa according to published protocols. The unsorted cells were plated in expansion medium as the control.

Reverse transcriptase-polymerase chain reaction (RT-PCR) analysis

Total RNA was isolated using Trizol (Invitrogen) as recommended by the manufacturer. First-strand cDNA was produced using Superscript II reverse transcriptase (Invitrogen) with Oligo dT. Primer sequences 
A

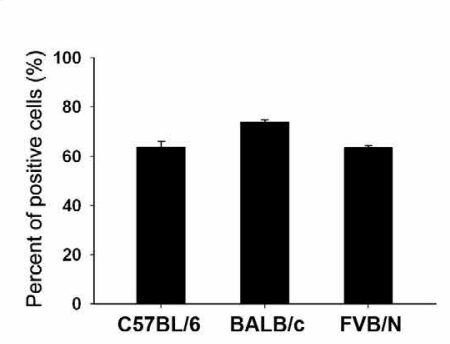

B

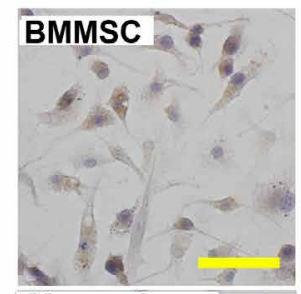

Macrophage

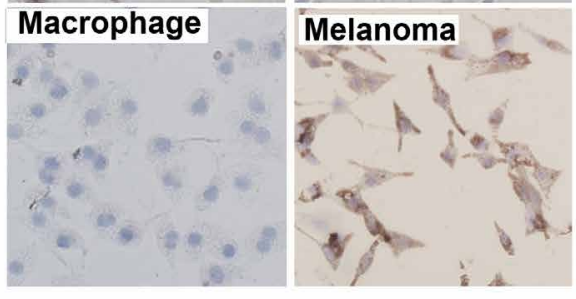

C

D
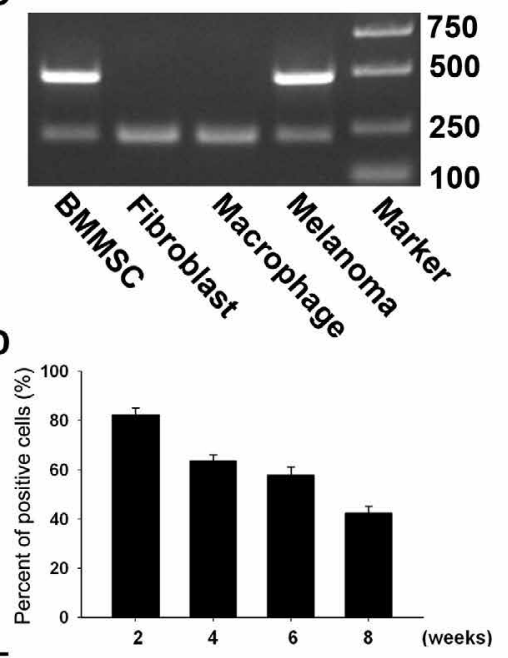

E

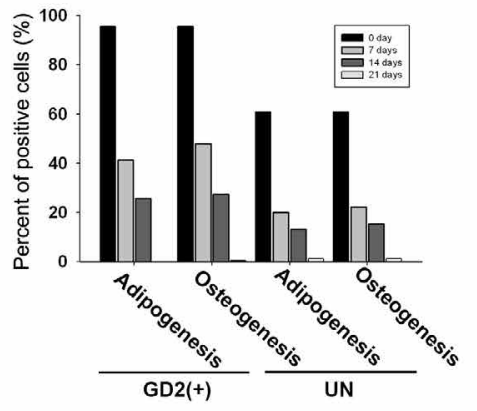

Fig. 1. GD2 expression on mBM-MSC. (A): Flow cytometric analysis of the expression of GD2 on mBM-MSC from 3 mouse strains: $\mathrm{C} 57 \mathrm{BL} / 6, \mathrm{BALB} / \mathrm{c}$ and $\mathrm{FVB} / \mathrm{N}, \mathrm{n}=3$ for P2. (B): Immunocytochemical staining of cultured mBM-MSC for GD2, in addition to fibroblast and macrophage. Staining of Melanoma cells served as a positive control, scale bar=100 $\mu \mathrm{m}$ (C): RT-PCR of total RNA isolated from mBM-MSC demonstrating the expression of GD2 synthase (474bp). No products expanded from total RNA of fibroblast and macrophage. PCR products of GD2 synthase in Melanoma cells served as a positive control. $\beta$-actin served as a control for the quality and quantity of RNA (200bp).(D): Change in percent GD2-positive cells with age. (E): The time course of GD2 expression during adipogenesis and osteogenesis. Abbreviations: UN, unsorted cells; GD2+, $\mathrm{GD2}{ }^{+}$-sorted cells.

and PCR conditions are described in Table 1. After electrophoresis on a 1.5\% agarose gel, the gel images of each PCR product were digitally captured with a CCD camera and analyzed with the NIH Imager beta version 2. Relative mRNA values for each gene in the semi-quantitative RT-PCR are presented as the ratio of the signal value of the specific PCR product to that of $\beta$-actin.

\section{Statistical analysis}

Data are presented as arithmetic mean \pm SD. Standard analyses were carried out using the Student t-test. Significant differences were considered those with a $P$ value of $<0.05$.

\section{Results}

GD2 expression on $\mathrm{mBM}-\mathrm{MSCS}$

Bone marrow cells were obtained from the femurs and tibias of mice by flushing the bone marrow and plated in tissue-culture flasks. After removal of non-adherent cells and 
Fig. 2. Flow cytometric analysis of unsorted, GD2+ and GD2-cells. The flow cytometric analysis of CD34, C-kit, CD45, CD11b, Sca-1, CD105, SSEA-1 and Nanog expression on unsorted, $\mathrm{GD}^{+}$and GD2 cells. Data represented mean \pm SD $(\mathrm{n}=3) .{ }^{*} p<0.05,{ }^{* *} p<0.01$. Abbreviations: UN, unsorted cells; GD2+, GD2+-sorted cells; GD2; GD2-sorted cells.

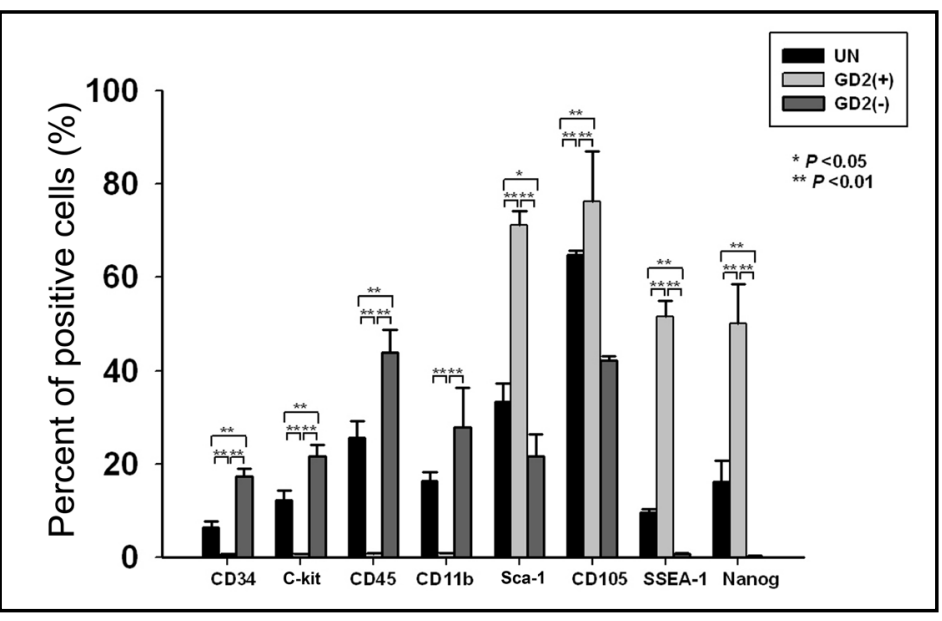

incubation for 2 weeks in growth medium, the cells with a spindle-shape characteristic of MSCs appeared to predominate in the cultures. Using the protocol described previously, we succeed in isolating mBM-MSCs from 3 mouse strains: C57BL/6, BALB/c and FVB/N. At passage 2, the culture-expanded cells were collected to assess GD2 expression by flow cytometry. The result showed that mBM-MSC from C57BL/6, BALB/c and FVB/N mouse all expressed high levels of GD2 $(63.66 \pm 2.47 \%, 73.88 \pm 0.81 \%$ and $63.44 \pm 1 \%$, respectively) (Fig. 1A). We further analyzed the expression of GD2 on mBM-MSCs from C57BL/6 mice by immunocytochemistry. Melanoma cells were chosen to serve as positive control. The immunocytochemical stain revealed that mBM-MSCs showed a pancellular expression of GD2, but fibroblasts and macrophages did not express this marker (Fig. 1B).These results were further supported by RT-PCR analysis which showed mBM-MSCs also expressed the mRNA for GD2 synthase, a key enzyme for GD2 biosynthesis, while fibroblasts and macrophages lacked GD2 synthase expression. 474 bp PCR products of GD2 synthase in melanoma cells served as a positive control (Fig. 1C). Importantly, flow cytometric analysis revealed that the expression level of GD2 showed a gradual decrease with the age of mice (Fig. 1D).Furthermore, we observed that the GD2 expression on mBM-MSCs gradually decreased along with the induction into osteoblasts or adipocytes (Fig. 1E).

\section{Flow cytometry analysis}

Using immunomagnetic beads, cultured mBM-MSCs from C57BL/6 mice were divided into GD2 ${ }^{+}$and GD2 cells. Then we investigated the expression of CD34, C-kit, CD45, CD11b, Sca-1 and CD105, which are cell surface markers associated with MSCs, on GD2+, GD2 ${ }^{-}$and unsorted cells. In addition, we observed the expression of SSEA-1 and Nanog, the embryonic stem cells markers, on these three fractions. The flow cytometric analysis showed that unsorted mBM-MSCs of the second passage still reacted with anti-CD34 $(6.49 \pm 1.27 \%)$, C-kit (12.26 $\pm 2.03 \%)$, CD $45(25.57 \pm 3.6 \%)$ and CD11b $(16.4 \pm 1.89 \%)$ mAbs. In contrast, GD2 ${ }^{+}$cells were negative for these hematopoietic antigens $(0.39 \pm 0.4 \%, 0.82 \pm 0.1 \%, 0.84 \pm 0.1 \%$ and $0.94 \pm 0.1 \%$, respectively). However, GD2 cells expressed significantly higher levels of the hematopoietic antigens: CD34 (17.26 $\pm 1.72 \%)$, C-kit $(21.73 \pm 2.43 \%)$, CD45 $(43.82 \pm 5.04 \%)$ and $\mathrm{CD} 11 \mathrm{~b}(27.91 \pm 8.38 \%)(p<0.01)$. Furthermore, the percentages of cells positive for anti-Sca-1 $(71.11 \pm 2.99 \%)$ and CD105 $(76.23 \pm 10.72 \%)$ in GD2 ${ }^{+}$cells were much higher than those in GD2 $(21.65 \pm 4.77 \%$ and $42.21 \pm 0.86 \%)$ and unsorted cells $(33.34 \pm 3.89 \%$ and $64.85 \pm 0.87 \%)(p<0.01)$. Notably, SSEA-1 and Nanog expression of GD2 ${ }^{+}$cells $(51.52 \pm 3.54 \%$ and $50.15 \pm 8.47 \%)$ was much higher than unsorted cells $(9.67 \pm 0.71 \%$ and $16.12 \pm 4.53 \%)$ but GD2 cells hardly expressed these markers $(0.85 \pm 0.11 \%$ and $0.23 \pm 0.14 \%)(p<0.01)$ (Fig. 2$)$.

\section{Clonogenicity and proliferation assays}

Our results showed that GD2 ${ }^{+}$cells displayed significantly more colony forming unitsfibroblasts (CFU-F) than unsorted cells in vitro. However, GD2 cells never showed any 
Fig. 3. Clonogenic and proliferative capacity of unsorted, GD2 ${ }^{+}$and GD2-cells. (A) $3 \times 10^{3}$ unsorted, GD2 ${ }^{+}$or GD2 cells were plated in growth medium in T-25 flasks for 14 days. For enumeration, the resulting colonies were stained with $3 \%$ Crystal violet. (B) Comparison of the different number of CFU-F colonies between three fractions. Data represented mean $\pm S D(n=3)$. ${ }^{* *} p<0.01$. (C) The proliferation rate of unsorted, $\mathrm{GD}^{+}$and GD2 cells was determined by cck- 8 . The viable cell numbers was determined every 3 days for 12 days. Data represented mean \pm SD (n=3), ${ }^{*} p<0.05,{ }^{* *} p<0.01,{ }^{*} p<0.01$. Abbreviations: CFU-F, colony forming unit -fibroblast; $\mathrm{UN}$, unsorted cells; GD2+, GD2+-sorted cells; GD2; GD2-sorted cells.

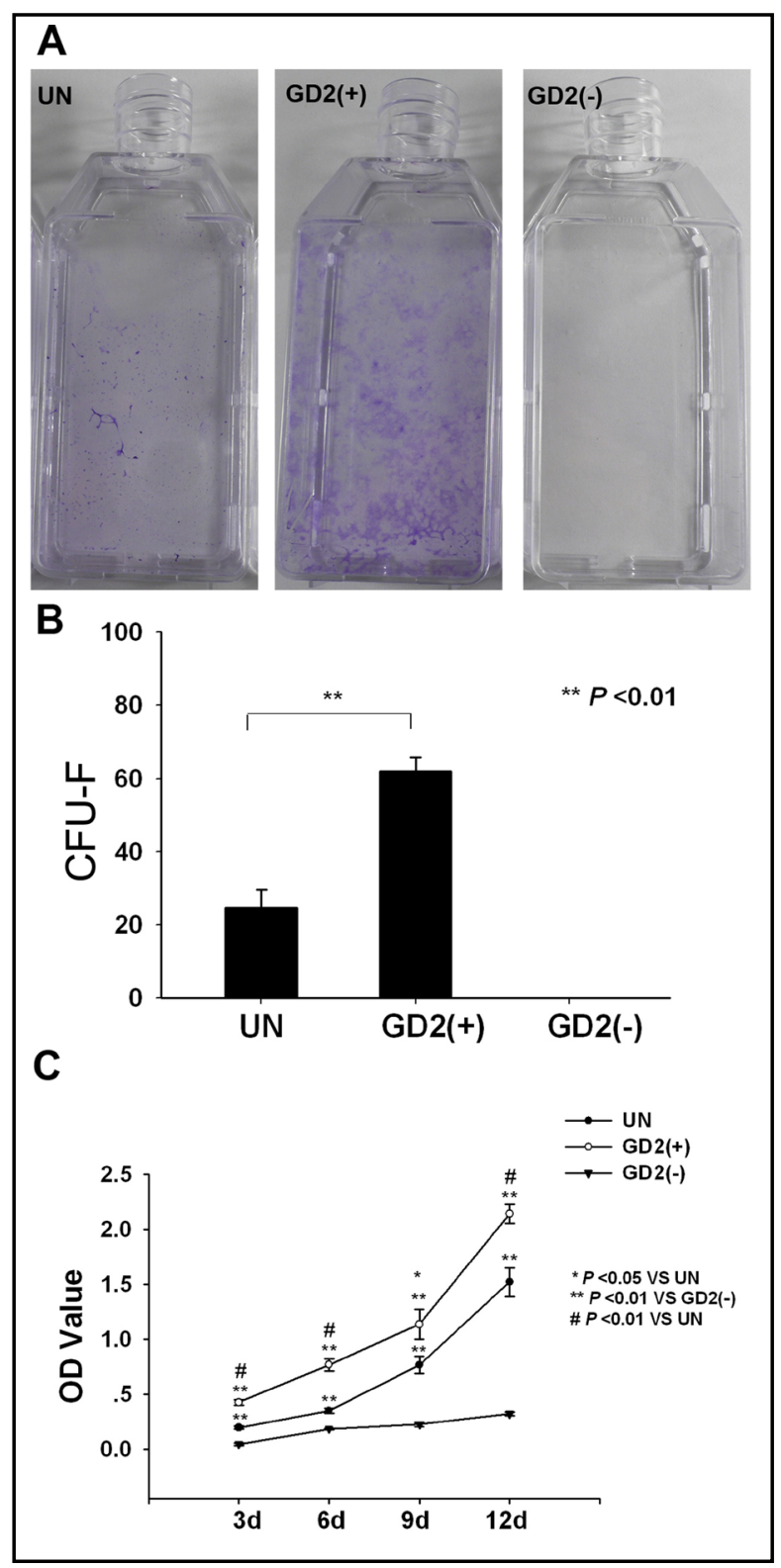

residual CFU-F activity (Fig. 3A). GD2 ${ }^{+}$cells yielded significantly higher number of CFU-F colonies $(61.97 \pm 3.85 \%)$ than unsorted cells $(24.65 \pm 4.88 \%)(p<0.01)$ (Fig. $3 B)$. In addition, GD2 ${ }^{+}$cells had a much higher proliferation rate compared to unsorted cells in 3, 6, 9 and 12 days $(p<0.01$ and $p<0.05)$. However, GD2 cells had a very low proliferation rate $(p<0.01)$ (Fig. 3C).

\section{Multi-lineage differentiation assays}

To compare the multi-lineage potential of GD2-sorted cells to unsorted cells, we cultured unsorted and GD2 ${ }^{+}$cells in adipogenic and osteogenic medium at the same density. After 21 days of induction, unsorted and GD2 ${ }^{+}$cells were stained positively with oil red 0 and von Kossa. However, lipid droplets and calcium deposits were not detected in the control groups. Moreover, oil red $\mathrm{O}$ and von Kossa staining showed that GD2 ${ }^{+}$cells were able to form adipocytes and osteoblasts more readily than unsorted cells (Fig. 4A). The results were supported by RT-PCR analysis which showed in two lineages GD2 ${ }^{+}$cells had higher gene expression than their parental cells but the control groups had no expression of these genes. 


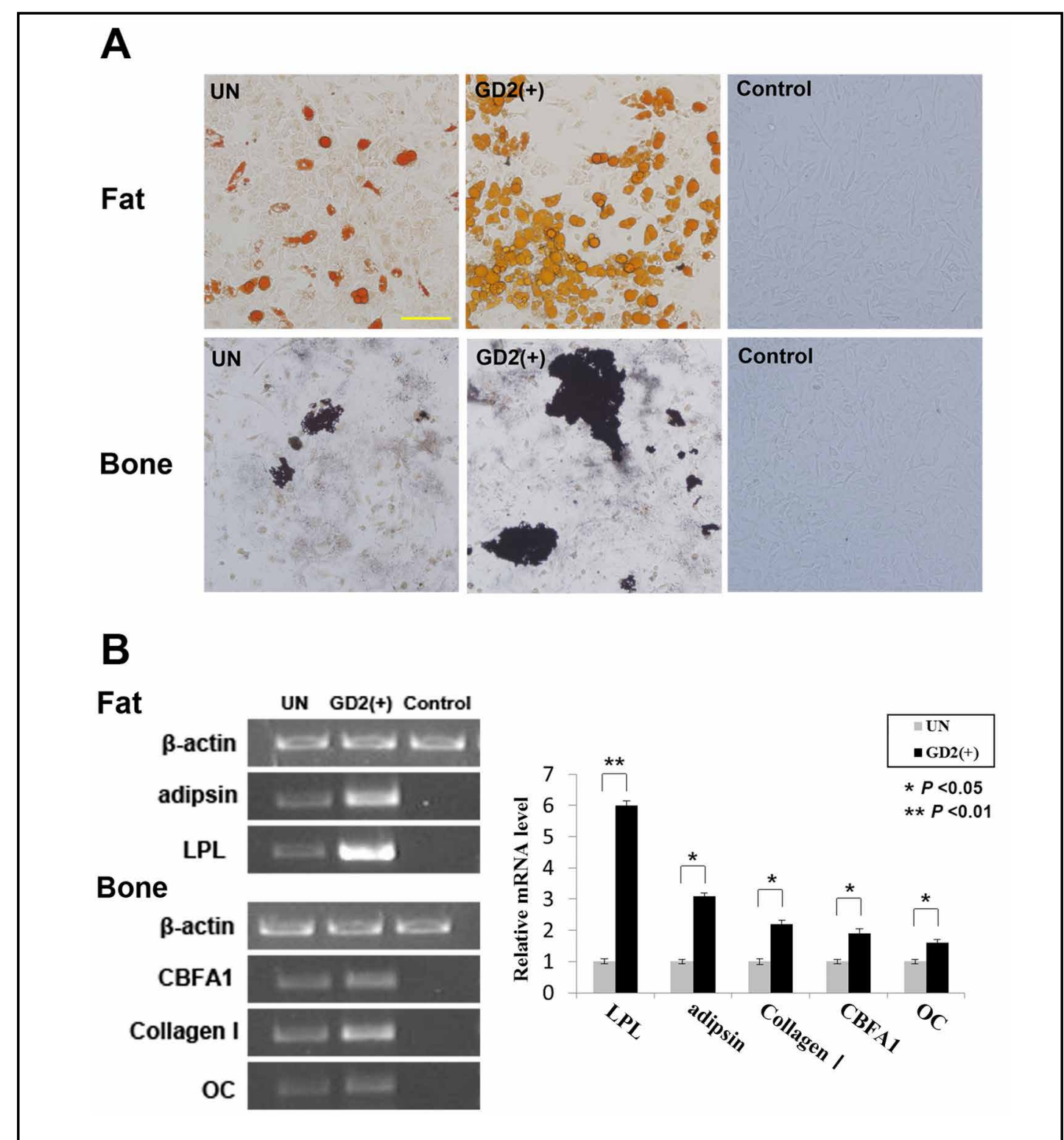

Fig. 4. Adipogenic and osteogenic differentiation capacity of unsorted and GD2+ cells. (A) Unsorted and $\mathrm{GD}^{+}$cells showed the different differentiation capability to adipocytes (Oil Red $\mathrm{O}$ stain) and osteoblasts (von Kossa stain). The unsorted cells were plated in expansion medium as the control. Scale bar $=100 \mu \mathrm{m}$. (B) Semi-quantification of mRNA levels of adipogenic and osteogenic specific genes in three groups. The adipogenic specific genes (Adipsin, LPL) and osteogenic specific genes (CBFA1, Collagen I, OC) were separated in $1.5 \%$ agarose gels (left). The relative value of each preparation is calculated by the gray numerical value of each specific product vs. that of $\beta$-actin (right). The average data of each preparation are evaluated based on three independent reactions and represented as mean \pm SD. ${ }^{*} p<0.05,{ }^{* *} p<0.01$ (right).Abbreviations: LPL, lipoprotein lipase; CBFA1, core-binding factor-1; OC, osteocalcin.

In adipogenesis, the expression of Adipsin and LPL in GD2 ${ }^{+}$cells was significantly higher than the parental cells ( 6 fold and 3 fold, respectively) $(p<0.01$ and $p<0.05)$. In contrast to their parental cells, CBFA1, Collage I and OC expression was rather high in induced GD2 ${ }^{+}$cells in osteogenesis (2.2 fold, 1.9 fold and 1.6 fold, respectively)( $p<0.05)$ (Fig. 4B). 
Xu et al.: mBM-MSCs Express the Neural Ganglioside GD2

\section{Discussion}

Despite their potential use in pre-clinical investigations, the identification and purification of murine MSCs have not been well developed. Compared to human MSCs, murine MSCs are far more difficult to be isolated from bone marrow due to the unwanted growth of non- MSCs in both primary and passage cultures [10-12]. Recently the report of a novel surface marker neural ganglioside GD2 distinguishing MSCs from other marrow elements [16] and our previous study demonstrating expression of GD2 in cultured UC-MSCs [17], have led us to consider that GD2 might be used as a maker for the identification and purification of murine BM-MSCs. To test this hypothesis, we used several experimental approaches to detect the expression of GD2 on mBM-MSCs, followed by further characterization of the cell populations sorted by GD2.

Our immunocytochemical and flow cytometric analysis revealed that GD2 was expressed on culture-expanded mBM-MSCs, and not on fibroblasts and macrophages. These results were further supported by RT-PCR analysis which showed expression of GD2 synthase, a key enzyme involved in GD2 synthesis, in mBM-MSCs but not in fibroblasts and macrophages. In addition, mBM-MSCs from various murine strains (C57BL/6, BALB/c and FVB/N) all expressed GD2. These results strongly suggested that the mBM-MSCs expressed GD2, which might be used to identify a homogenous population of MSCs within murine bone marrow.

We sorted both GD2 ${ }^{+}$and GD2- fractions from mBM-MSCs cultures based on GD2 expression. As previously reported [13-15], the flow cytometric analysis showed that unsorted mBM-MSCs of second passage expressed hematopoietic antigens CD45 and CD11b or progenitor/stem cell antigens CD34 and C-kit, indicating existence of hematopoietic cells in this population. However, these surface markers were not present in GD2 ${ }^{+}$fractions. In contrast, GD2 fractions expressed high levels of these antigens. These results indicated that hematopoietic cells could be eliminated from bone marrow cultures in early passages by GD2 immunosorting. Tropel et al. found that even after eliminating granulo-monocytic cells using CD11b antibody, such cells continued to exist in the culture one week after immunodepletion [15]. We found that Sca-1 expression on GD2+ fractions was higher than unsorted fractions, which is in agreement with previous reports demonstrating expression of Sca-1 on cultured murine MSCs $[13,14,21]$. Moreover, we observed that both the GD2 ${ }^{+}$and GD2- fractions expressed CD105, suggesting that this marker alone was not sufficient to distinguish MSCs from other cell populations. It has been reported that MSCs present a similar cell surface phenotype to terminally differentiated fibroblasts, including expression of CD105 [22, 23].

Notably, the GD2+ fractions maintained a higher level of SSEA-1, an embryonic stem cells marker [24], which was reported to define the most primitive progenitors in the adult murine bone marrow mesenchymal compartment [25], compared to the unsorted and GD2fractions. Meanwhile the GD2+ fractions expressed a much higher level of Nanog, a key transcription factor for the maintenance of the undifferentiated state of ES cells [26], in comparison to the unsorted and GD2 fractions. Furthermore, we observed that GD2 expression gradually decreased with age and disappeared once mBM-MSCs had differentiated into osteoblasts or adipocytes. Taken together, our results strongly suggested that GD2 likely identifies an early population of MSCs in murine bone marrow. This hypothesis was further supported by the following results. First, the results showed that the GD2 ${ }^{+}$cells displayed a significantly higher number of CFU-F colonies as well as a higher proliferation rate compared to the unsorted cells. The GD2-sorted cells failed to grow. Second, there was a significant difference in the differentiation potential between the GD2 ${ }^{+}$and unsorted cells. Oil Red $\mathrm{O}$ and von Kossa staining revealed that GD2 ${ }^{+}$cells were able to form adipocytes and osteoblasts more readily than the parental cells. Consistent with these observations, there was a significant up-regulation of adipogenic and osteogenic specific genes in the induced GD2 ${ }^{+}$cells. Collectively, these studies suggested that GD2 marked the early precursor cells of mBM-MSCs.

In conclusion, our studies demonstrate that GD2 identifies a primitive subpopulation of mBM-MSCs and that it can be used as a novel marker for isolating murine MSCs from bone 
marrow with high specificity. We propose that GD2 may be a conserved marker for MSCs from various mammalian species in addition to humans. Our findings may have a significant impact on the studies of isolation and purification of mBM-MSCs as well as help in better understanding the biology of these cells.

\section{Acknowledgments}

This study was supported by National Natural Science Foundation of China (81201686), 863 projects from Ministry Science \& Technology of China (2006AA02A110) and National Natural Science Foundation of China (30570357 and 30600238).

\section{References}

1 Pittenger MF, Mackay AM, Beck SC, Jaiswal RK, Douglas R, Mosca JD, Moorman MA, Simonetti DW, Craig S, Marshak DR: Multilineage potential of adult human mesenchymal stem cells. Science 1999;84:143-147.

-2 Sanchez-Ramos J, Song S, Cardozo-Pelaez F, Hazzi C, Stedeford T, Willing A, Freeman TB, Saporta S, Janssen W, Patel N, Cooper DR, Sanberg PR: Adult bone marrow stromal cells differentiate into neural cells. Exp Neurol 2000;164:247-256.

- Colter DC, Sekiya I, Prockop DJ: Identification of a subpopulation of rapidly self-renewing and multipotential adult stem cells in colonies of human marrow stromal cells. Proc Natl Acad Sci U S A 2001;98:7841-7845.

- 4 Horwitz EM, Gordon PL, Koo WK, Marx JC, Neel MD, McNall RY, Muul L, Hofmann T: Isolated allogeneic bone marrow-derived mesenchymal cells engraft and stimulate growth in children with osteogenesis imperfecta: implications for cell therapy of bone. Proc Natl Acad Sci U S A 2002;99:8932-8937.

-5 Hofstetter CP, Schwarz EJ, Hess D, Widenfalk J, El Manira A, Prockop DJ, Olson L: Marrow stromal cells form guiding strands in the injured spinal cord and promote recovery. Proc Natl Acad Sci USA 2002;99:21992204.

6 Lee RH, Seo MJ, Reger RL, Spees JL, Pulin AA, Olson SD, Prockop DJ: Multipotent stromal cells from human marrow home to and promote repair of pancreatic islets and renal glomeruli in diabetic NOD/scid mice. Proc Natl Acad Sci U S A 2006;103:17438-17443.

-7 Aurich I, Mueller LP, Aurich H, Luetzkendorf J, Tisljar K, Dollinger MM, Schormann W, Walldorf J, Hengstler JG, Fleig WE, Christ B: Functional integration of hepatocytes derived from human mesenchymal stem cells into mouse livers. Gut 2007;56:405-415.

8 Ohtaki H, Ylostalo JH, Foraker JE, Robinson AP, Reger RL, Shioda S, Prockop DJ: Stem/progenitor cells from bone marrow decrease neuronal death in global ischemia by modulation of inflammatory/immune responses. Proc Natl Acad Sci USA 2008;105:14638-14643.

-9 Friedenstein AJ, Chailakhyan RK, Latsinik NV, Panasyuk AF, Keiliss-Borok IV: Stromal cells responsible for transferring the microenvironment of the hemopoietic tissues. Cloning in vitro and retransplantation in vivo. Transplantation 1974;17:331-340.

10 Phinney DG, Kopen G., Isaacson RL, Prockop DJ: Plastic adherent stromal cells from the bone marrow of commonly used strains of inbred mice: variations in yield, growth, and differentiation. J Cell Biochem 1999;2:570-585.

11 Meirelles Lda S, Nardi NB: Murine marrow-derived mesenchymal stem cell: isolation, in vitro expansion, and characterization. Br J Haematol 2003;23:702-711.

12 Peister A, Mellad JA, Larson BL, Hall BM, Gibson LF, Prockop DJ: Adult stem cells from bone marrow (MSCs) isolated from different strains of inbred mice vary in surface epitopes, rates of proliferation, and differentiation potential. Blood 2004;103:1662-1668.

13 Sun S, Guo Z, Xiao X, Liu B, Liu X, Tang PH, Mao N: Isolation of mouse marrow mesenchymal progenitors by a novel and reliable method. Stem Cells 2003;21:527-535. 
Xu et al.: mBM-MSCs Express the Neural Ganglioside GD2

14 Baddoo M, Hill K, Wilkinson R, Gaupp D, Hughes C, Kopen GC, Phinney DG: Characterization of mesenchymal stem cells isolated from murine bone marrow by negative selection. J Cell Biochem 2003;89:1235-1249.

15 Tropel P, Noël D, Platet N, Legrand P, Benabid AL, Berger F: Isolation and characterisation of mesenchymal stem cells from adult mouse bone marrow. Exp Cell Res 2004;295:395-406.

-16 Martinez C, Hofmann TJ, Marino R, Dominici M, Horwitz EM: Human bone marrow mesenchymal stromal cells express the neural ganglioside GD2: a novel surface marker for the identification of MSCs. Blood 2007;109:4245-4248.

-17 Xu J, Liao W, Gu D, Liang L, Liu M, Du W, Liu P, Zhang L, Lu S, Dong C, Zhou B, Han Z: Neural ganglioside GD2 identifies a subpopulation of mesenchymal stem cells in umbilical cord. Cell Physiol Biochem 2009;23:415424.

18 Kopen GC, Prockop DJ, Phinney DG: Marrow stromal cells migrate throughout forebrain and cerebellum, and they differentiate into astrocytes after injection into neonatal mouse brains. Proc Natl Acad Sci U S A 1999;96:10711-10716.

19 Nagai Y, Akashi S, Nagafuku M, Ogata M, Iwakura Y, Akira S, Kitamura T, Kosugi A, Kimoto M, Miyake K: Essential role of MD-2 in LPS responsiveness and TLR4 distribution. Nat Immunol 2002;3:667-672.

20 Cheo DL, Ruven HJ, Meira LB, Hammer RE, Burns DK, Tappe NJ, van Zeeland AA, Mullenders LH, Friedberg EC: Characterization of defective nucleotide excision repair in XPC mutant mice. Mutat Res 1997;74:1-9.

21 Van Vlasselaer P, Falla N, Snoeck H, Mathieu E : Characterization and purification of osteogenic cells from murine bone marrow by two-color cell sorting using anti-Sca-1 monoclonal antibody and wheat germ agglutinin. Blood 1994;84:753-763.

22 Barry FP, Boynton RE, Haynesworth S, Murphy JM, Zaia J: The monoclonal antibody SH-2, raised against human mesenchymal stem cells, recognizes an epitope on endoglin (CD105). Biochem Biophys Res Commun 1999;265:134-139.

23 Wagner W, Wein F, Seckinger A, Frankhauser M, Wirkner U, Krause U, Blake J, Schwager C, Eckstein V, Ansorge W, Ho AD: Comparative characteristics of mesenchymal stem cells from human bone marrow, adipose tissue, and umbilical cord blood. Exp Hematol 2005;33:1402-1416.

24 Henderson JK, Draper JS, Baillie HS, Fishel S, Thomson JA, Moore H, Andrews PW: Preimplantation human embryos and embryonic stem cells show comparable expression of stage-specific embryonic antigens. Stem Cells 2002;20:329-337.

-25 Anjos-Afonso F, Bonnet D: Nonhematopoietic/endothelial SSEA-1+ cells define the most primitive progenitors in the adult murine bone marrow mesenchymal compartment. Blood 2007;109:1298-1306.

-26 Mitsui K, Tokuzawa Y, Itoh H, Segawa K, Murakami M, Takahashi K, Maruyama M, Maeda M, Yamanaka S: The homeoprotein Nanog is required for maintenance of pluripotency in mouse epiblast and ES cells. Cell 2003;113:631-642. 